\title{
ARTICLE Resveratrol and STAT inhibitor enhance autophagy in ovarian cancer cells
}

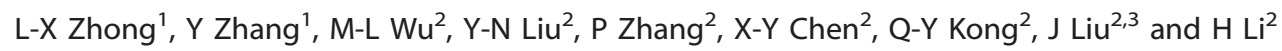

\begin{abstract}
Autophagic activity reflects cellular response to drug treatment and can be regulated by STAT3 signaling. Resveratrol inhibits STAT3 activation and causes remarkable growth arrest and cell death of ovarian cancer (OC) cells. However, the autophagic status and its relevance with resveratrol's anti-OC effects remain unclear. We analyzed the states of autophagic activities, the nature of autophagosomes and the levels of autophagy-related proteins (LC-3, Beclin 1 and STAT3) in resveratrol-treated CAOV-3 and OVCAR-3 OC cells using multiple approaches. We elucidated the correlation of STAT3 inhibition with autophagic activity by treating OC cells with an upstream inhibitor of STAT proteins, AG490. Resveratrol efficiently suppressed growth, induced apoptosis and inactivated STAT3 signaling of the two OC cell lines. We found enhanced autophagic activity accompanied with Beclin-1 upregulation and LC3 enzymatic cleavage in resveratrol-treated OC cells. Immunofluorescent (IF) microscopic and IF-based confocal examinations demonstrated the accumulation of cytoplasmic granules co-labeled with LC3 and cytochrome C in resveratrol- or AG490-treated OC cells. Using electron microscopy, we confirmed an increase in autophagosomes and mitochondrial spheroids in either resveratrol- or AG490-treated OC cells. This study demonstrates the abilities of resveratrol to enhance apoptotic and autophagic activities in OC cells, presumably via inactivating STAT3 signaling. Resveratrol or the selective JAK2 inhibitor also leads to mitochondrial turnover, which would be unfavorable for OC cell survival and sensitize OC cells to resveratrol.
\end{abstract}

Cell Death Discovery (2016) 2, 15071; doi:10.1038/cddiscovery.2015.71; published online 25 January 2016

\section{INTRODUCTION}

Ovarian cancer (OC) is one of the commonest female malignancies with an extremely poor prognosis. ${ }^{1-3}$ Surgical treatment is the first choice for removing OCs in cases that are well-differentiated, relatively small, or confined to the ovary. ${ }^{4,5}$ Unfortunately, the majority of OC patients (75\%) are diagnosed at advanced stages because of the subtle symptoms at the early stages of ovarian carcinogenesis. ${ }^{6}$ Consequently, most OC patients die of metastases due to peritoneal transplantation or blood stream spreading. ${ }^{7}$ Therefore, adjuvant chemotherapy is required to prevent tumor relapse and dissemination. ${ }^{8}$

Although more accurate staging of the disease and more aggressive surgical excision of tumor spots in the abdomen have somewhat improved therapeutic outcomes, the overall survival rates continue to lack promise. ${ }^{9}$ Furthermore, drug resistance often occurs among OC patients and severe toxic effects caused by conventional anticancer drugs greatly reduce patients' quality of life..$^{9-11}$ It is therefore urgent to explore more effective and less toxic agents with clearer molecular targets for better adjuvant management of OCs.

It has been increasingly recognized that resveratrol (3,5,4'-trihydroxy-trans-stilbene) possesses cancer-preventive and -suppressive activities. ${ }^{12-14}$ More importantly, resveratrol has little cytotoxic effect on normal tissues in vitro and in vivo at effective anticancer doses, reflecting its potential value in cancer treatments when administered appropriately. ${ }^{12,15}$ Resveratrol exerts its anti-OC effects by altering multiple molecular targets ${ }^{16,17}$ and regulating apoptotic and autophagic activities. ${ }^{18}$ For instance, the activated Wnt, Notch and STAT3 signaling pathways in human
OVCAR-3 and CAOV-3 cells are concurrently inhibited, of which STAT3 inactivation seems a critical molecular event because selective inhibition of STAT3 signaling leads cancer cells to apoptosis. ${ }^{19}$ However, STAT3 signaling has repressive roles in autophagy of cancer cells with different biological consequences. $^{20}$ For instance, STAT3 inhibits autophagy and pancreatic cancer cell growth by downregulating LC3 expression, ${ }^{21}$ whereas inhibition of this signaling suppresses growth and promotes autophagy and apoptosis of esophageal squamous cell carcinoma cells. ${ }^{22}$ These data suggest that the interplay of STAT3 signaling and autophagy, and its biological consequences to cancer cells, may vary by cell type. To date, no studies have addressed the role of STAT3 in regulating autophagic activity in OC cells and the impact of resveratrol on that process. The current study thus aims to address the above issues, using human OVCAR-3 and CAOV-3 as the experimental models because their growth and STAT3 activation can be concurrently suppressed by resveratrol. ${ }^{19}$

\section{RESULTS}

Resveratrol suppressed OC cell growth

$\mathrm{H} / \mathrm{E}$ staining showed distinct morphological alterations in resveratrol-treated populations (Figure 1a) and terminal deoxynucleotide transferase (TdT)-mediated dUTP-biotin nick-end labeling (TUNEL) assay demonstrated that TUNEL-positive cells appeared in the two cell lines after being treated by resveratrol for $24 \mathrm{~h}$ and becomes more popular at $48 \mathrm{~h}$ time point (Figure 1a). The viable/unviable cell fractionation and MTT assay revealed that

\footnotetext{
${ }^{1}$ Department of Clinical Oncology, Second Affiliated Hospital of Dalian Medical University, Dalian 110042 , China; ${ }^{2}$ Liaoning Laboratory of Cancer Genetics and Epigenetics,

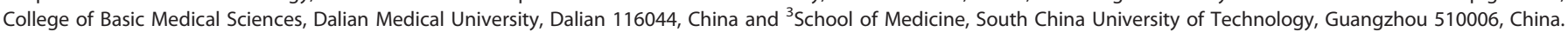
Correspondence: J Liu (jialiudl@aliyun.com) or H Li (lihongmcn@dlmedu.edu.cn)

Received 22 October 2015; revised 25 November 2015; accepted 4 December 2015; Edited by N Barlev
} 
the growth of all three OC cells was significantly suppressed $(P<0.01)$ by $100 \mu \mathrm{M}$ resveratrol in time-related pattern in comparison with that of their normally cultured counterparts (Figures $1 \mathrm{~b}$ and c). Ki-67-positive cells were greatly reduced after $48 \mathrm{~h}$ of resveratrol exposure (Figure $1 \mathrm{~d}$ ).
Inactivated STAT3 signaling in resveratrol-suppressed OC cells Immunocytochemical staining demonstrated that p-STAT3 was distributed in either cytoplasm or the nuclei of the two OC cell lines, which became reduced in cytoplasm and rarely translocalized into nuclei after resveratrol treatment (Figure 2a). The results a

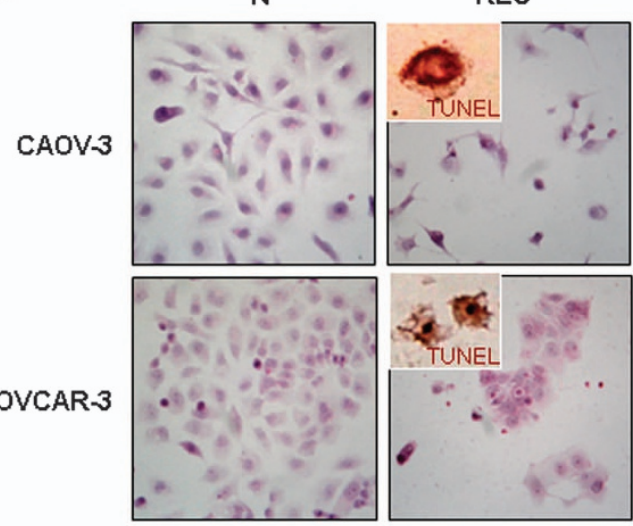

C

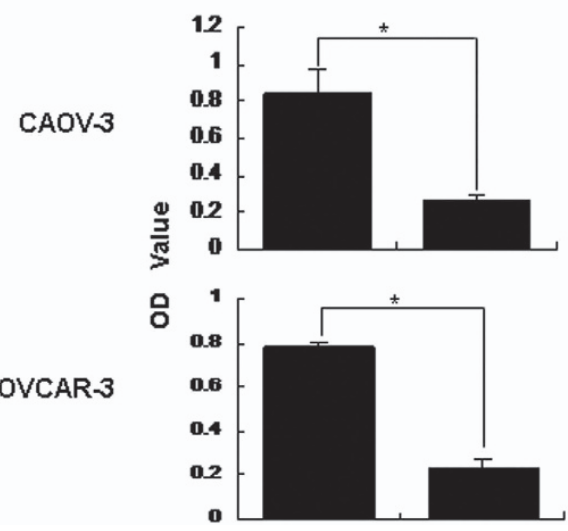

b

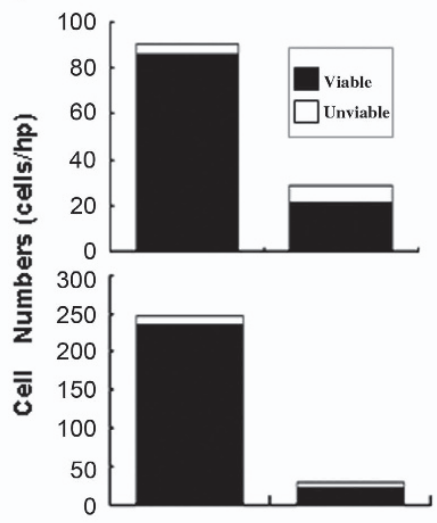

$\mathrm{Ki}-67$
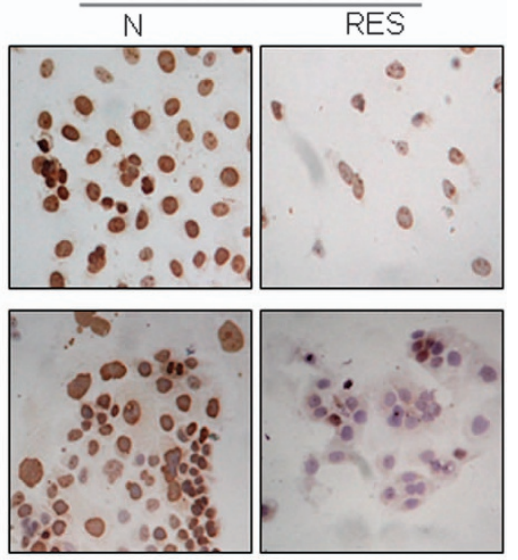

Figure 1. Cellular responses of human ovarian cancer CAOV-3 and OVCAR-3 cells to resveratrol. (a) Hematoxylin and eosin morphological staining and TUNEL staining performed on CAOV-3 and cells without (N) and with treatment with $120 \mu \mathrm{M}$ resveratrol for $48 \mathrm{~h}$ (RES). (b) Viable/ unviable cell counting of CAOV-3 and OVCAR-3 cells treated by resveratrol for $48 \mathrm{~h}$. (c) Evaluation of the responses of CAOV-3 and OVCAR-3 cells to resveratrol by MTT cell proliferation assay. (d) Ki-67-oriented immunocytochemical staining performed on CAOV-3 and OVCAR-3 cells without and with resveratrol treatment.

a
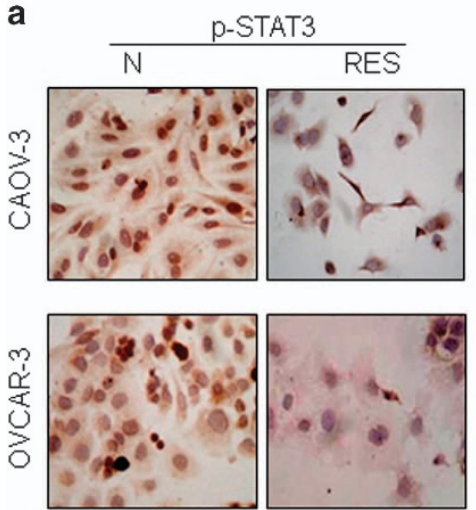

b

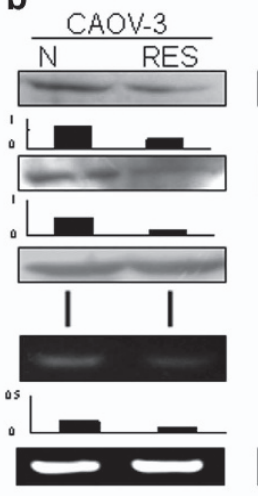

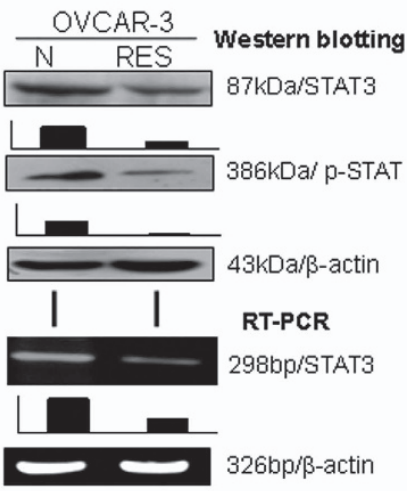

Figure 2. Statuses of STAT3 activation in CAOV-3 and OVCAR-3 cells without and with $120 \mu \mathrm{M}$ resveratrol treatment. (a) Immunocytochemical illustration of p-STAT3 intracellular distribution patterns in the two human ovarian cancer cell lines without (N) and with (RES) resveratrol treatment. (b) Western blot and RT-PCR analyses of STAT3 and p-STAT3 levels in the two human ovarian cancer cell lines without (N) and with (RES) resveratrol treatment. 
a

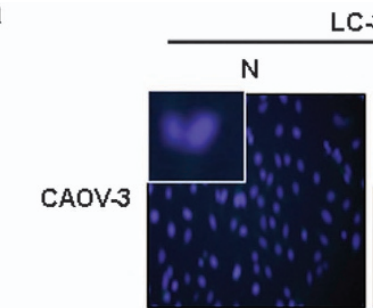

LC.3

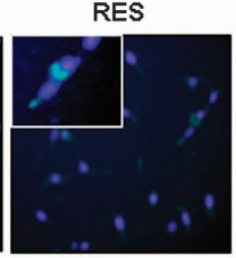

OVCAR 3
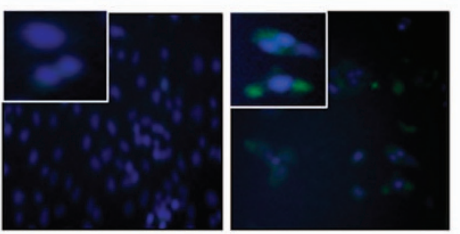

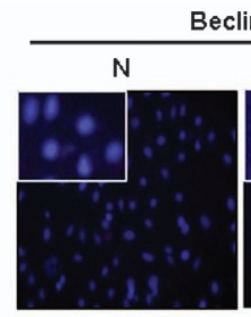

Beclin1
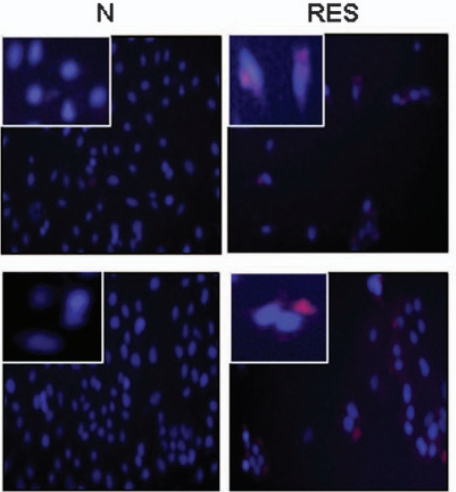

b
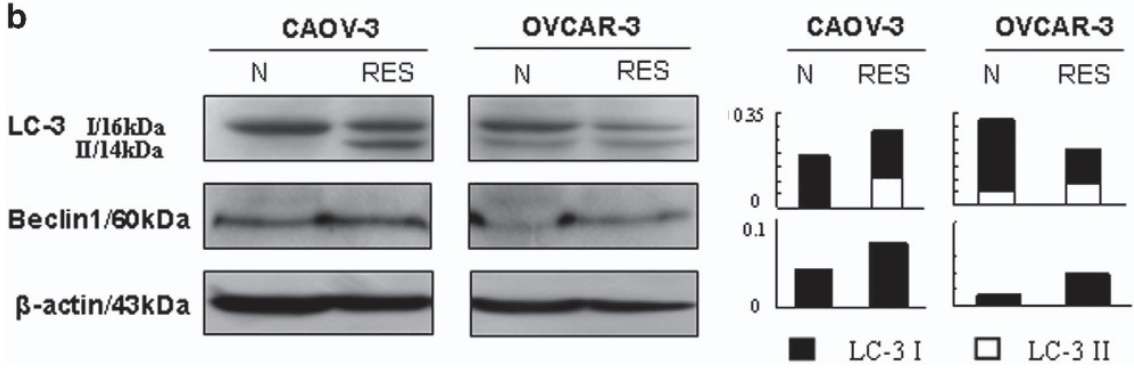

Figure 3. Evaluation of LC-3 and Beclin 1 expression in CAOV-3 and OVCAR-3 cells without (N) and with resveratrol treatment (RES) by immunofluorescent staining (a) and western blotting (b).

of western blotting were in agreement with ICC findings in terms of reduced level of p-STAT3 and the decreased production of total STAT3 proteins (Figure 2b). RT-PCR showed distinct downregulation of STAT3 transcription in resveratrol-treated OVCAR-3 and CAOV-3 cells (Figure $2 \mathrm{c}$ ).

Resveratrol-enhanced autophagic activities

To elucidate the status of autophagy in OC cells and its relevance with resveratrol-caused cell crisis, double immunoflorescent staining for LC3 and Beclin-1 was conducted on the cell-bearing coverslips, which showed that these two proteins were weakly labeled in normally cultured $O C$ cells and became enhanced especially for LC3 after being treated by $120 \mu \mathrm{M}$ resveratrol for $48 \mathrm{~h}$ (Figure $3 \mathrm{a}$ ). The following $\mathrm{H} / \mathrm{E}$ staining performed on the coverslips with double immunoflorescent staining revealed distinct cell death in resveratrol-treated populations (insets of Figure 3a).

Resveratrol altered LC3 and Beclin-1 expression

The autophagic activities can be reflected by the levels of autophagy-associated protein LC3 and Beclin- 1 and the presence of $14 \mathrm{kDa}$ LC3-II, a proteolytic form of LC3. ${ }^{23}$ Therefore, the statuses of LC3 and Beclin-1 expression in the two OC cell lines without and with drug treatment were examined by western blotting. The results showed that the levels of LC3 and Beclin-1 were elevated in resveratrol-treated $\mathrm{OC}$ cells and an additional 14 kDa LC3-II band appeared in CAOV-3 and the increased fraction of this band in OVCAR-3 cells after $48 \mathrm{~h}$ of resveratrol treatment (Figure 3b).

Negative correlation of STAT3 activation and Beclin-1 expression Immunocytochemical staining with p-STAT3 antibody revealed that distinct nuclear translocation of STAT3 was commonly observed in normally cultured CAOV-3 and OVCAR-3 cells, which became weakened or diminished in the cells treated by $100 \mu \mathrm{M}$ resveratrol for $48 \mathrm{~h}$ (Figure 2a). In contrast, Beclin-1 was expressed in low levels in CAOV-3 and OVCAR-3 cells under normal culture condition and was upregulated upon resveratrol treatment (Figure 3b).

LC3+/cytochrome C+ granules in resveratrol-treated cells LC3 is a commonly used marker to monitor autophagosomes and cytochrome $C$ proteins are preferably localized in mitochondria. ${ }^{24}$ To identify the feature(s) of autophagic bodies, LC3 and cytochrome $\mathrm{C}$ double IF staining combined with confocal laser scanning microscope observation were conducted on the OC cells without and with resveratrol treatment. The results demonstrate that the conventional IF image of normally cultured OC cells was weakly green in the cytoplasm and the confocal one showed the predominant distribution of granules labeled with LC3 (green) or cytochrome C (red; Figure 4a). The IF staining patterns were apparently altered in resveratrol-treated cells by showing the color transition of IF images from weakly green to strong orange and yellow at $24 \mathrm{~h}$ to green and yellow at $48 \mathrm{~h}$ and to yellow and green at $72 \mathrm{~h}$ time points. In agreement with the IF microscopic findings, the confocal images demonstrated the appearance of $\mathrm{LC}^{+} /$cytochrome $\mathrm{C}^{+}$granules in the cytoplasm of resveratroltreated cells, especially those treated for $24 \mathrm{~h}$ (Figure $4 \mathrm{~b}$ ).

\section{AG490 induced autophagosis and apoptosis}

To elucidate the role of STAT3 signaling in regulating autophagic activity, AG490, a selective JAK2 inhibitor, was used to treat the CAOV-3 cell line for $48 \mathrm{~h}$, followed by TUNEL apoptotic cell labeling, STAT3-oriented immunocytochemical staining and double immunoflorescent staining/IF for LC3/ Becline-1 and LC3/cytochrome C. As shown in Figure 5, AG490 suppressed cell growth, inhibited STAT3 nuclear translocation and upregulated Becline-1 and, especially, LC3 expression. 

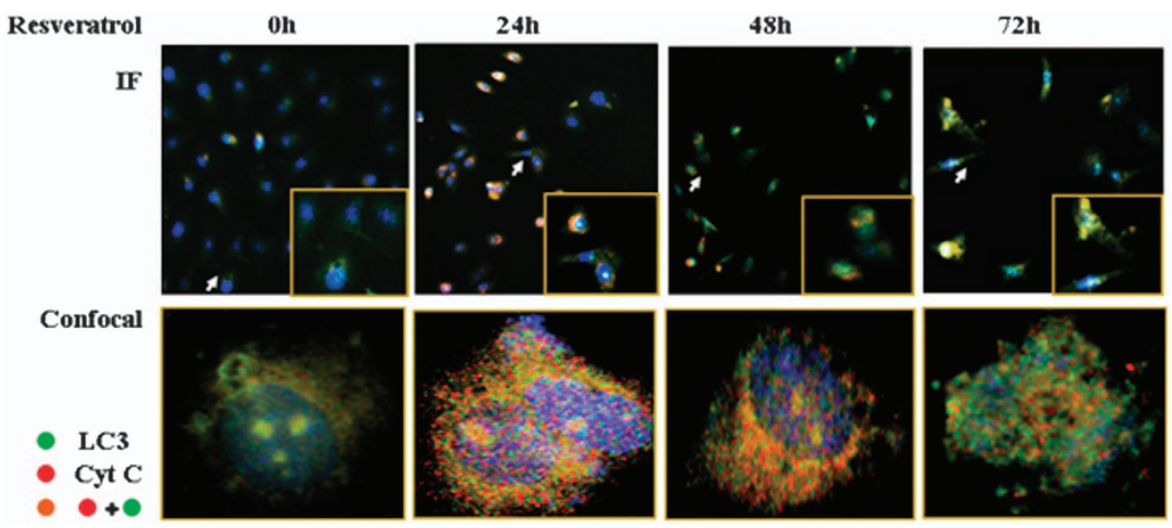

Figure 4. Double LC3 (green) and cytochrome C (red) immunofluorescent labeling of CAOV-3 cells treated by resveratrol at different time points. IF, the images taken under conventional IF microscope. Confocal, the images taken under confocal laser scanning microscope.

a

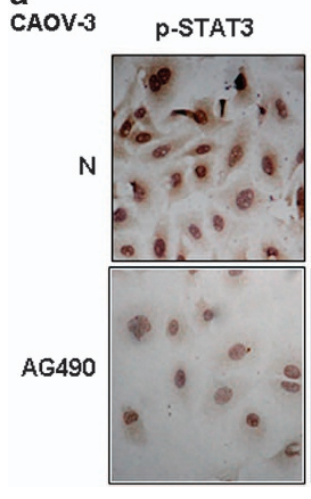

LC3 3

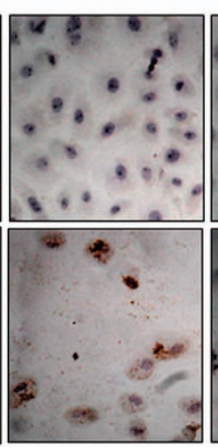

b

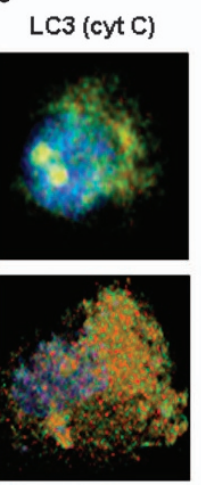

C

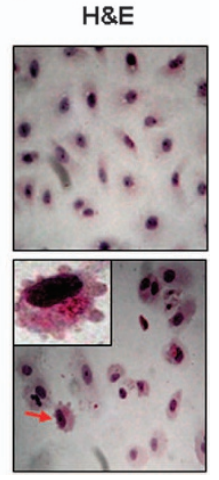

Figure 5. Evaluation of STAT3 activation, autophagic activities and apoptosis of CAOV-3 cells without (N) and with AG490 treatment (AG490). (a) Immunocytochemical staining for p-STAT3, LC-3 and Beclin 1; (b) Confocal images of double LC-3 and cytochrome C IF labeling; (c) H/E morphological staining performed on the IF stained coverslips for confocal examination. Arrow indicates the cell shown in the inset with higher magnification.

Furthermore, LC3+/cytochrome C+ granules (Figure 5c) and TUNEL-positive cells (Figure $5 b$ ) were frequently observed in AG490-treated CAOV-3 cells.

Ultrastructural illustration of resveratrol increased autophagosomes

Electron microscopic graphs clearly demonstrated the abundance of cytosolic vacuoles enclosed with membrane bounded components in resveratrol- and AG490-treated OC cells rather than their normally cultured counterparts (Figure 6). It was also found that those vacuoles were not identical in terms of their sizes and contents (Figure 6b). As shown in the images in higher magnifications, some vacuoles were relatively small and involved the formation of a single outer membrane (left); in contrast, the others were larger, in a ring- or C-shaped morphology, defined by a double membrane and rich in criesta-like structures (right).

\section{DISCUSSION}

Increasing evidence demonstrates the promising inhibitory effects of resveratrol on OC cells. ${ }^{19,25,26}$ Our recent results show that Wnt, Notch and STAT3 signaling pathways are activated in human OC OVCAR-3 and CAOV-3 cells, which are concurrently inhibited by resveratrol, accompanied with remarkable growth arrest and cell death. ${ }^{19}$ Our previous study also indicates that STAT3 signaling is critical for the growth and survival of OC cells and is the major molecular target of resveratrol because STAT3 transcription is downregulated in resveratrol-treated OC cells. Further, the selective inhibition of STAT, but not the Wnt or Notch pathways, leads to a similar biological consequence caused by resveratrol, including the increased fractions of apoptosis. ${ }^{19}$ Nevertheless, it is still unclear whether apoptosis is the only death program triggered by resveratrol and inactivation of STAT3 signaling in OC cells, or whether additional survival or death mechanisms are altered as well.

Autophagy is a basic cellular maintenance mechanism by which unnecessary or dysfunctional cellular components are degraded and cellular survival can be maintained in response to stress. ${ }^{27}$ In the context of cancers, the roles of autophagy seem diverse; it occurs as an adaptive or protective response to environmental alterations, ${ }^{28}$ or it appears to commit the cells to programmed cell death type II. ${ }^{29}$ Although the relevance of autophagic activity with therapeutic efficacies of anticancer drugs has been discussed, the conclusions contradict. For instance, enhanced autophagocytosis has been found in resveratrol-sensitive OC cells, ${ }^{18,30}$ while it has a prosurvival role in resveratrol-induced cytotoxicity in human U251 glioblastoma cells. ${ }^{31}$ In agreement with Lang's findings, ${ }^{30}$ we observed enhanced autophagic activity, together with distinct growth arrest and remarkable apoptosis, in OVCAR-3 and CAOV-3 cells treated by resveratrol for $48 \mathrm{~h}$. In addition to the above cellular crisis, we found that resveratrol upregulates two autophagy-related genes, LC3 and Beclin-1. LC3 II generated by 
Control

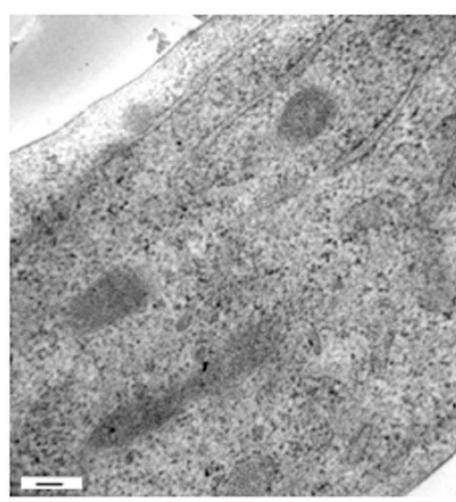

$\overline{100 \mathrm{~nm}}$

$\mathrm{HV}=120.0 \mathrm{kV}$

Direct Mag: $80000 x$

AMT Camera system
Resveratrol
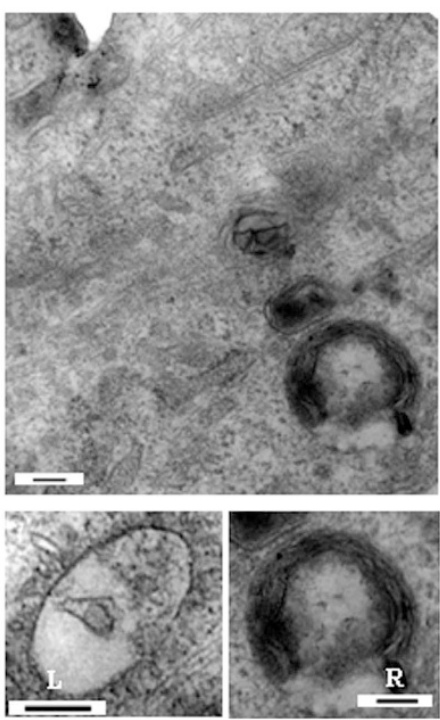

AG490
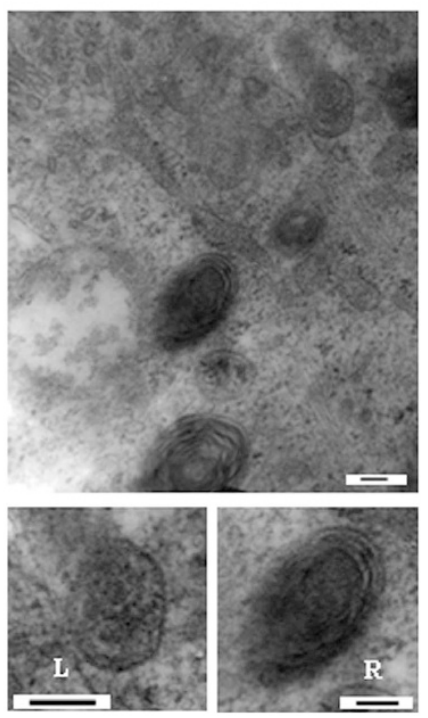

Figure 6. Electron microscopic illustration of autophagic statuses of CAOV-3 cells cultured normally (Control) and treated by resveratrol or AG490 for $48 \mathrm{~h}$. The single membrane autophagosomes and the double membrane defined mitochondrial spheroids found in resveratrol- and AG490-treated cells are shown, respectively, with higher magnifications in the left (L) and the right (R) small images.

enzymatic cleavage indicates the initiation of autophagy. ${ }^{23}$ Our western blotting results clearly demonstrate the appearance of an additional LC3 II band and a twofold increase in the level of LC3II in resveratrol-treated OVCAR3 cells. These results suggest that resveratrol enhances autophagic activity of OC cells when exerting its anti-OC effects. Because the activated autophagocytosis is maintained throughout the treatment and is overlapped with apoptosis, this cellular event may reflect continuous turnover of intracellular components, which would be harmful for cell growth and maintenance.

STAT3 signaling is the critical survival factor of OC cells ${ }^{19}$ and the main molecular target of resveratrol. ${ }^{12,19,32}$ Although reduction of STAT3 expression can induce mitochondrial dysfunction and autophagy in mouse cardiac $\mathrm{HL}-1$ cells, ${ }^{33}$ the involvement of this signaling pathway in regulating autophagic activities of cancer cells requires further study. Because of the co-existence of STAT3 inactivation and enhanced autophagic activity in resveratrol-treated OC cells, we elucidated the potential correlation of these two biological events by inhibiting STAT3 signaling with AG490, followed by LC3 immunocytochemistry staining. Similar to our findings from the resveratrol-treated cells, the nuclear translocation of p-STAT3 was largely inhibited, accompanied by enhanced LC3 and Beclin 1 expression and remarkable apoptotic cell death in AG490-treated OC cells. These findings thus indicate the importance of activated STAT3 signaling in regulating the autophagic activity of OC cells. Alternatively, the enhanced autophagic activity in resveratrol-treated OC cells may be, at least in part, the consequence of STAT3 inactivation.

Autophagocytosis occurs in several ways to degrade unnecessary or damaged intracellular elements. For instance, the autophagosomes that contain defective mitochondria are called mitochondrial autophagy or mitophagy. ${ }^{34}$ Although the enhanced autophagic activity has been found in resveratrol-treated OC cells with suppressed STAT3 signaling, it is worthwhile to know the type of autophagy happening in those cells because it may reflect the pattern and the extent of intracellular turnover caused by resveratrol and the STAT3 selective inhibitor. Cytochrome C, a small hemeprotein, appears on the surface of the inner mitochondrial membrane and acts as an essential component of the electron transport chain. ${ }^{35}$ Therefore, it can be used as a parameter to identify mitochondria. ${ }^{36}$ To determine the nature of LC3+ granules in resveratrol-treated OC cells and the cells treated by the STAT-selective inhibitor AG490, we performed double IF staining combined with confocal examination on those cells using a green-labeled mouse anti-human LC3 antibody and a redlabeled rabbit anti-human cytochrome $\mathrm{C}$ antibody. Merging of the LC3 and cytochrome C images changed many intracellular granules to orange-yellow, indicating they are positive for both LC3 and cytochrome $C$ and contain a mitochondrial component. In contrast, LC3+/cytochrome C+ granules are uncommon in the OC cells cultured under normal conditions. Electron microscopic images further demonstrate the presence of two types of structural alterations of membrane-bounded compartments in resveratrol- or AG490-treated OC cells. One is defined by a single membrane and contains membrane elements in its lumen, the typical phenotype of autophagosome. ${ }^{37}$ The other is considered a mitochondrial spheroid because of its double membranesurrounded ball-like structure with an internal compartment opening to the external cytoplasmic space. ${ }^{38}$ These phenomena suggest for the first time the abilities of resveratrol to increase overall autophagic activities and to cause extensive mitochondrial turnover. Because AG490-treated cells show the similar IF labeling pattern and ultrastructural phenotype, resveratrol-promoted autophagocytosis may be largely due to STAT3 inactivation.

Currently, the therapeutic implication of enhanced autophagic activity in cancer cells has not been well clarified. ${ }^{39}$ Because remarkable autophagocytosis overlaps with extensive apoptosis in resveratrol-treated $\mathrm{OC}$ cells, it is possible that resveratrol increases the pressure of intracellular damages, leading to enhanced autophagic elimination of the damaged elements, severe loss of organelle function and finally cell death. ${ }^{40}$ This supposition is supported by the presence of mitochondrial spheroid formations in resveratrol-treated OC cells, because this unique mitochondrial structure can be induced by mitochondrial toxin and evolved in the pathological conditions related to mitochondrial injury. ${ }^{41}$ So far, the relevance of mitochondrial spheroids with autophagy has not yet been ascertained. The findings of increased LC3+/cytochrome C+ granules and mitochondrial spheroids in resveratrol-suppressed $\mathrm{OC}$ cells indicate that the mitochondrial dysfunction and exhaustion may make OC cells more fragile to 
drug treatment. The existence of mitochondrial spheroids in AG490-treated cells implicates the importance of STAT signaling for maintaining mitochondrial integrity. ${ }^{42}$ However, this speculation should be further elucidated by determining whether the autophagic turnover and cell crisis can be rescued by prevention of resveratrol-caused STAT3 inactivation or reactivation of STAT3 downstream signaling in resveratrol-treated OC cells.

Taken together, our current study demonstrates resveratrol's abilities to promote autophagy and apoptosis and to cause mitochondrial turnover in human $\mathrm{OC}$ cells. This further confirms the therapeutic value of this nontoxic compound in the adjuvant management of OCs. The extremely low bioavailability of orally administered resveratrol has long been the major obstacle to clinical use of the compound. According to our findings from rat orthotopic glioblastoma and bladder cancer models, ${ }^{12,15}$ this can be largely overcome by organ-oriented administration approaches. In this context, intraperitoneal injection, a conventional way to treat abdominal malignancies, ${ }^{43}$ would be a potential option for practical treatment of OCs with resveratrol.

\section{CONCLUSIONS}

This study demonstrates the abilities of resveratrol to promote autophagic and apoptotic activities and to inactivate STAT3 signaling in human OC cells. This nontoxic compound would therefore be of therapeutic value in the adjuvant management of OCs when administered appropriately.

\section{MATERIALS AND METHODS}

Cell culture and treatment

Human OC CAOV-3 cells ${ }^{44}$ were cultured in Dulbecco's modified Eagle's essential medium containing $12 \%$ fetal bovine serum (Gibco Life Science, Grand Island, NY, USA) under $37^{\circ} \mathrm{C}$ and $5 \% \mathrm{CO}_{2}$ conditions and human $\mathrm{OC}$ OVCAR-3 cells ${ }^{45}$ in RPMI 1640 under $37^{\circ} \mathrm{C}$ and $5 \% \mathrm{CO}_{2}$ condition. The cells $\left(5 \times 10^{4} / \mathrm{ml}\right)$ were plated to culture dishes (NUNC, Denmark) and incubated for $24 \mathrm{~h}$ before the experiments. Trans-resveratrol (Sigma Chemical Co., St. Louis, MO, USA) was dissolved in DMSO (Sigma) to a stock concentration of $100 \mathrm{mM}$, wrapped in aluminum foil for protection against light and stored at $-20^{\circ} \mathrm{C}$. The cells were exposed to $120 \mu \mathrm{M}$ Res for $72 \mathrm{~h}$ (72 h). The cells cultured under normal condition and treated by $0.2 \%$ DMSO were used as normal and background controls, respectively. Cell numbers and viabilities were checked in $24 \mathrm{~h}$ intervals. The cell-bearing coverslips collected from the three experimental groups were fixed in cold acetone or $4 \%$ paraformaldehyde $(\mathrm{pH} 7.4)$ for morphological and immunocytochemical examinations. The experimental groups were set in triplicate and the experiments were repeated at least for three times to establish confidential conclusion.

\section{Evaluation of cell growth}

Haematoxylin and eosin $(\mathrm{H} / \mathrm{E})$ staining was performed on the cell-bearing coverslips to evaluate the morphological features of the three OC cell lines with different treatments. The effects of resveratrol on cell proliferation were determined by 3-[4,5-Dimethylthiazol-2-yl]-2,5-diphenyl-tetrazolium bromide (MTT) assay ${ }^{17}$ and shown in $\mathrm{OD}$ values. The fractions of viable and unviable cells were estimated with cell counting apparatus (TC20 Automated Cell Counter, BIO RAD). TUNEL assay was employed to detect apoptotic cells according to producer's instructions (Promega Corporation, USA).

\section{Sequential coverslip collection from resveratrol-treated} populations

It has been proposed that altered autophagic activity is the early cellular response to drug treatment ${ }^{46}$ and has certain relevance with chemosensitivities of the treated cells. ${ }^{46-48}$ NEST-DISH, a joint product of NEST Biotech Inc. Wuxi and BioArrayTech Inc. Dalian, China (China Patent for Invention: ZL200610047607.0), is the novel coverslip preparation dish, from which 32 cell-bearing coverslips can be prepared and then collected sequentially or simultaneously for different research purposes. ${ }^{49}$ Therefore, this novel device was adopted for sequential analyses of the statuses of autophagic activities and STAT3 signaling of resveratrol-treated cells by collecting several pieces of cell-bearing coverslips at 4 time points $(0,24$, 48 and 72 h) of resveratrol treatment. The coverslips collected were fixed properly for H/E morphological staining, LC3 and Becline-1 double immunofluorescent staining, STAT3-oriented immunocytochemical staining and, when necessary, TUNEL apoptotic cell labeling.

\section{Immunocytochemical staining}

Immunocytochemical staining (ICC) was performed on the cell-bearing coverslips collected regularly from different experimental groups by the method described previously. ${ }^{18}$ The antibodies against human STAT3 and p-STAT3 were purchased from Santa Cruz Biotechnology, Inc, CA. Color reaction was developed using 3, 3'-diaminobenzidine tetrahydrochloride (DAB). According to the labeling intensity, the staining results were evaluated by two independent researchers and scored as negative $(-)$ if no immunolabeling was observed in target cells, weakly positive $(+)$ if the labeling was faint, moderately positive $(++)$, and strongly positive $(>++)$ when the labeling was stronger or distinctly stronger than $(++)$.

\section{Immunofluorescent labeling and observation}

For immunofluorescent staining (IF), the cell-bearing coverslips were rinsed with phosphate-buffered solution (PBS; $\mathrm{pH}$ 7.4) for 3 times, blocked with $10 \%$ goat serum in PBS ( $\mathrm{pH}$ 7.4) for 20 min, incubated overnight with primary antibody against target protein (LC3 or Becline-1) and finally co-incubated with fluorescence-labeled goat anti-rabbit and rabbit antimouse IgG ( 1 : 200; Santa Cruz Biotechnology, Inc) at $37^{\circ} \mathrm{C}$ for $60 \mathrm{~min}$ in darkness. The nuclei were labeled by DAPI (blue fluorescence). The coverslips were observed and photographed under a fluorescence microscope (BX51, Olympus, Japan) and a confocal laser scanning fluorescent microscopy (SP8, Leica, Heidelberg, Germany).

\section{RNA isolation and RT-PCR}

Sample RNAs were isolated from the two human OC cell lines without and with resveratrol treatment for $48 \mathrm{~h}$. By the method described elsewhere, ${ }^{8}$ reverse transcription (RT) was performed on RNA samples, followed by polymerase chain reaction (PCR) with a pair of primers specific for the CDNA of an individual gene (STAT3: Forward primer: 5'-GGGTGGAGAAG GACATCAGCGGTAA-3', reverse primer: 5'-GCCGACAATACTTTCCGAATGC-3'; $\beta$-actin: forward primer: $5^{\prime}$-GCATGGAGTCCTGTGGCAT-3', reverse primer: $5^{\prime}$-CTAGAAGCA TITGCGGTGG-3'19). The PCR products were resolved on $1 \%$ agarose gel containing ethidium bromide $(0.5 \mu \mathrm{g} / \mathrm{ml})$, visualized and photographed using UVP Biospectrum Imaging System (UVP, Inc, Upland, CA). The $\beta$-actin PCR products generated from the same RT solution were cited as quantitative controls.

\section{Protein preparation and western blotting}

Total cellular proteins were prepared from the cells under different culture conditions. The sample proteins $(50 \mu \mathrm{g} /$ well) were separated in $10 \%$ sodium dodecylsulfate-polyacrylamide gel electrophoresis and transferred to polyvinylidene difluoride membrane (Amersham, Buckinghamshire, UK). The membrane was blocked with $5 \%$ skimmed milk in TBS-T $(10 \mathrm{mM}$ Tris- $\mathrm{HCl}, \mathrm{pH} 8.0,150 \mathrm{mM} \mathrm{NaCl}$ and $0.5 \%$ Tween 20 ) at $4{ }^{\circ} \mathrm{C}$, rinsed $10 \mathrm{~min}$ for three times with TBS-T, followed by $3 \mathrm{~h}$ incubation at room temperature with the first antibody and then $1 \mathrm{~h}$ incubation with HRP-conjugated antimouse or anti-rabbit IgG (Zymed Lab, Inc). The bound antibody was detected using the enhanced chemiluminescence system (Roche $\mathrm{GmbH}$, Mannheim, Germany). After removing the labeling signal by incubation with stripping buffer, ${ }^{8}$ the membrane was reprobed with other antibodies one by one until all of the parameters were examined.

\section{Autophagosome identification and quantification}

LC3 acts downstream from the ATG5-ATG12 system and distributes on the outer and inner surfaces of the autophagosome. ${ }^{50}$ Cytochrome $C, a$ component of the electron transport chain, is found loosely associated with the inner membrane of the mitochondrion. ${ }^{35}$ Therefore, these two proteins were employed as the biomarkers for identifying the nature(s) of the increased autophagosomes in resveratrol-treated OC cells by double IF staining. The criteria of the judgment were as follows: the intracellular LC3-positive green granules under fluorescent microscope indicated the presence of autophagosomes; the cytochrome C-positive red granules indicated that they were mitochondria or contained mitochondrial 
elements; the granules co-labeled by LC3 and Cytochrome C suggested that they were mitochondrial autophagosomes. The LC3 and Cytochrome $C$ labeling patterns of OC cells without and with resveratrol or AG490 treatment were visualized in the forms of conventional IF microscopic and confocal IF microscopic images.

\section{Treatment with AG490 selective JAK2 inhibitor}

AG490 has been regarded an upstream STAT inhibitor. ${ }^{51}$ To elucidate the influence of STAT inhibition in autophagic activity, this compound in the working concentration of $80 \mu \mathrm{M}$ was therefore used to treat the three OC cells growing on the coverslips for $48 \mathrm{~h}$. The coverslips collected were subjected to H\&E morphological staining, Beclin-1 and p-STAT3 immunocytochemical staining and double immunoflorescent staining using rabbit anti-human LC3- and mouse anti-human cytochrome $\mathrm{C}$ antibodies.

\section{Electron microscopic examination}

The cell-bearing coverslips were washed with PBS for three times (10 min/time) and then fixed in $2.5 \%$ glutaraldehyde $(30 \mathrm{~min}, 50 \mathrm{mM}$ cacodylate buffer, $\mathrm{pH} 7.2$ ) and $2 \% \mathrm{OsO}_{4}$ (30 mim, same buffer). Ultra-thin sections $(0.1 \mu \mathrm{M})$ were prepared and examined under a Philips CM100 transmission electron microscope (FEI Company, USA). Images were captured by charge-coupled device camera equipped with TCL-EM-Menu version 3 from Tietz Video and Image Processing Systems (Gaunting, GmbH, Friedrichshafen, Germany) as described elsewhere. ${ }^{29}$ The coverslips bearing the cells without resveratrol or AG490 treatment were used as controls.

\section{Statistical analysis}

Statistical analyses were performed using the software of SPSS 17.0 (IBM San Francisco, CA, USA). Data were given as the means \pm S.D. Statistical analyses were performed using one-way ANOVA and $P<0.05$ were considered to indicate significance.

\section{ACKNOWLEDGEMENTS}

This work was supported by the grants from National Natural Science Foundation of China (No. 81450016, 81272786, 81071971, 81072063 and 30971038), Research Fund for PhD supervisors from National Education Department of China (20122105110005), Program Fund for Liaoning Excellent Talents in University (LU2012078) and Program for Changjiang Scholar and Innovative Research Team in University (PCSIRT). The funders had no role in study design, data collection and analysis, decision to publish, or preparation of the manuscript.

\section{AUTHOR CONTRIBUTIONS}

ZLX carried out the analyses of cellular and molecular effects of resveratrol on ovarian cancer cells. ZY participated in the data analyses and morphological examination. MLW carried out cell culture and the statistical analysis. LYN and ZP participated in sample preparation for protein and RNA analyses. LC carried out western blotting and RT-PCR analyses. CXY and KQY participated in immunocytochemical staining. $\mathrm{JL}$ and $\mathrm{LH}$ involved in the design of the study and performed the statistical analysis. LH participated in the design of the study and coordination and helped to draft the manuscript. All authors read and approved the final manuscript.

\section{COMPETING INTERESTS}

The authors declare no conflict of interest.

\section{REFERENCES}

1 Lowe KA, Chia VM, Taylor A, O'Malley C, Kelsh M, Mohamed M et al. An international assessment of ovarian cancer incidence and mortality. Gynecol Oncol 2013; 130: 107-114.

2 Usui A, Ko SY, Barengo N, Naora H. P-cadherin promotes ovarian cancer dissemination through tumor cell aggregation and tumor-peritoneum interactions. Mol Cancer Res 2014; 12: 504-513.

3 Gujar S, Dielschneider R, Clements D, Helson E, Shmulevitz M, Marcato P et al. Multifaceted therapeutic targeting of ovarian peritoneal carcinomatosis through virus-induced immunomodulation. Mol Ther 2013; 21: 338-347.
4 Collinson F, Qian W, Fossati R, Lissoni A, Williams C, Parmar M et al. ICON1 collaborators. Optimal treatment of early-stage ovarian cancer. Ann Oncol 2014; 25: $1165-1171$.

5 Signorelli M, Fruscio R, Ceppi L, Dell'anna T, Vitobello D, Chiappa V et al. The role of pelvic and aortic lymphadenectomy at second look surgery in apparent early stage ovarian cancer after inadequate surgical staging followed by adjuvant chemotherapy. Gynecol Oncol 2014; 132: 312-315.

6 Husseinzadeh N. Status of tumor markers in epithelial ovarian cancer has there been any progress? A review. Gynecol Oncol 2011; 120: 152-157.

7 Deng J, Wang L, Chen H, Li L, Ma Y, Ni J et al. The role of tumour-associated MUC1 in epithelial ovarian cancer metastasis and progression. Cancer Metastasis Rev 2013; 32: 535-551.

8 Stoeckle E, Bourdarias L, Guyon F, Croce S, Brouste V, Thomas L et al. Progress in survival outcomes in patients with advanced ovarian cancer treated by neo-adjuvant platinum/taxane-based chemotherapy and late interval debulking surgery. Ann Surg Oncol 2014; 21: 629-636.

9 Della Pepa C, Tonini G, Santini D, Losito S, Pisano C, Di Napoli M et al. Low grade serous ovarian carcinoma: from the molecular characterization to the best therapeutic strategy. Cancer Treat Rev 2015; 41: 136-143.

10 Davis A, Tinker AV, Friedlander M. 'Platinum resistant' ovarian cancer: What is it, who to treat and how to measure benefit? Gynecol Oncol 2014; 133: 624-631.

11 Galluzzi L, Vitale I, Michels J, Brenner C, Szabadkai G, Harel-Bellan A et al. Systems biology of cisplatin resistance: past, present and future. Cell Death Dis 2014; 5: e1257.

$12 \mathrm{Wu} \mathrm{ML}$, Li H, Yu LJ, Chen XY, Kong QY, Song $\mathrm{X}$ et al. Short-term resveratrol exposure causes in vitro and in vivo growth inhibition and apoptosis of bladder cancer cells. PLoS One 2014; 9: e89806.

13 Sun Z, Li H, Shu XH, Shi H, Chen XY, Kong QY et al. Distinct sulfonation activities in resveratrol-sensitive and resveratrol-insensitive human glioblastoma cells. FEBS $J$ 2012; 279: 2381-2392.

14 Shu XH, Li H, Sun Z, Wu ML, Ma JX, Wang JM et al. Identification of metabolic pattern and bioactive form of resveratrol in human medulloblastoma cells. Biochem Pharmacol 2010; 79: 1516-1525.

15 Shu XH, Wang LL, Li H, Song X, Shi S, Gu JY et al. Diffusion efficiency and bioavailability of resveratrol administered to rat brain by different routes: therapeutic implications. Neurotherapeutics 2015; 12: 491-501.

16 Dann JM, Sykes PH, Mason DR, Evans JJ. Regulation of vascular endothelial growth factor in endometrial tumour cells by resveratrol and EGCG. Gynecol Oncol 2009; 113: $374-378$

17 Vergara D, Simeone $\mathrm{P}$, Toraldo D, Del Boccio P, Vergaro V, Leporatti S et al. Resveratrol downregulates Akt/GSK and ERK signalling pathways in OVCAR-3 ovarian cancer cells. Mol Biosyst 2012; 8: 1078-1087.

18 Opipari Jr AW, Tan L, Boitano AE, Sorenson DR, Aurora A, Liu JR. Resveratrol-induced autophagocytosis in ovarian cancer cells. Cancer Res 2004; 64: 696-703.

19 Zhong LX, Li H, Wu ML, Liu XY, Zhong MJ, Chen XY et al. Inhibition of STAT3 signaling as critical molecular event in resveratrol-suppressed ovarian cancer cells. J Ovarian Res 2015; 8: 25.

20 Yuan X, Du J, Hua S, Zhang H, Gu C, Wang J et al. Suppression of autophagy augments the radiosensitizing effects of STAT3 inhibition on human glioma cells. Exp Cell Res 2015; 330: 267-276.

21 Gong J, Muñoz AR, Chan D, Ghosh R, Kumar AP. STAT3 down regulates LC3 to inhibit autophagy and pancreatic cancer cell growth. Oncotarget 2014; 5: 2529-2541.

22 Feng $\mathrm{Y}$, Ke C, Tang Q, Dong H, Zheng X, Lin W et al. Metformin promotes autophagy and apoptosis in esophageal squamous cell carcinoma by downregulating Stat3 signaling. Cell Death Dis 2014; 5: e1088.

23 Kadowaki M, Karim MR. Cytosolic LC3 ratio as a quantitative index of macroautophagy. Methods Enzymol 2009; 452: 199-213.

24 Babbitt SE, Sutherland MC, Francisco BS, Mendez DL, Kranz RG. Mitochondrial cytochrome c biogenesis: no longer an enigma. Trends Biochem Sci 2015; 40: 446-455.

25 Farrand L, Byun S, Kim JY, Im-Aram A, Lee J, Lim S et al. Piceatannol enhances cisplatin sensitivity in ovarian cancer via modulation of $\mathrm{p} 53, \mathrm{X}$-linked inhibitor of apoptosis protein (XIAP), and mitochondrial fission. J Biol Chem 2013; 288: 23740-23750.

26 Mikuła-Pietrasik J, Sosińska P, Książek K. Resveratrol inhibits ovarian cancer cell adhesion to peritoneal mesothelium in vitro by modulating the production of a5 $\beta 1$ integrins and hyaluronic acid. Gynecol Oncol 2014; 134: 624-630.

27 Schiavi A, Maglioni S, Palikaras K, Shaik A, Strappazzon F, Brinkmann V et al. Iron-starvation-induced mitophagy mediates lifespan extension upon mitochondrial stress in C. elegans. Curr Biol 2015; 25: 1810-1822.

28 Chen P, Hu T, Liang Y, Jiang Y, Pan Y, Li C et al. Synergistic inhibition of autophagy and neddylation pathways as a novel therapeutic approach for targeting liver cancer. Oncotarget 2015; 6: 9002-9017. 
29 Kana U, Toshihiko A, Funakoshi T, Hashimoto K, Uemura K. Extrusion of mitochondrial contents from lipopolysaccharide-stimulated cells: Involvement of autophagy. Autophagy 2015; 11: 1520-1536.

30 Lang F, Qin Z, Li F, Zhang H, Fang Z, Hao E. Apoptotic cell death induced by resveratrol is partially mediated by the autophagy pathway in human ovarian cancer cells. PLoS ONE 2015; 10: e0129196.

$31 \mathrm{Li} \mathrm{J,} \mathrm{Qin} \mathrm{Z,} \mathrm{Liang} \mathrm{Z.} \mathrm{The} \mathrm{prosurvival} \mathrm{role} \mathrm{of} \mathrm{autophagy} \mathrm{in} \mathrm{resveratrol-induced}$ cytotoxicity in human U251 glioma cells. BMC Cancer 2009; 9: 215.

32 Zhang P, Li H, Yang B, Yang F, Zhang LL, Kong QY et al. Biological significance and therapeutic implication of resveratrol-inhibited Wnt, Notch and STAT3 signaling in cervical cancer cells. Genes Cancer 2014; 5: 154-164.

33 Elschami M, Scherr M, Philippens B, Gerardy-Schahn R. Reduction of STAT3 expression induces mitochondrial dysfunction and autophagy in cardiac HL-1 cells. Eur J Cell Biol 2013; 92: 21-29.

34 Patergnani S, Pinton P. Mitophagy and mitochondrial balance. Methods $\mathrm{Mol}$ Biol 2015; 1241: 181-194.

35 Aluri HS, Simpson DC, Allegood JC, Hu Y, Szczepanek K, Gronert S et al. Electron flow into cytochrome c coupled with reactive oxygen species from the electron transport chain converts cytochrome $\mathrm{c}$ to a cardiolipin peroxidase: role during ischemia-reperfusion. Biochim Biophys Acta 2012; 1840: 3199-3207.

36 Chen TT, Tian X, Liu CL, Ge J, Chu X, Li Y. Fluorescence activation imaging of cytochrome $\mathrm{c}$ released from mitochondria using aptameric nanosensor. J Am Chem Soc 2015; 137: 982-989.

37 Eskelinen EL, Reggiori F, Baba M, Kovács AL, Seglen PO. Seeing is believing: the impact of electron microscopy on autophagy research. Autophagy 2015; 7: 935-956.

38 Ding WX, Li M, Biazik JM, Morgan DG, Ni HM, Goheen M et al. Electron microscopic analysis of a spherical mitochondrial structure. J Biol Chem. 2012; 287: 42373-42378.

39 Lindqvist LM, Simon AK, Baehrecke EH. Current questions and possible controversies in autophagy. Cell Death Discovery 2015; 1: 15036.

40 Chang SH, Hwang CS, Yin JH, Chen S, Yang DI. Oncostatin M-dependent Mcl-1 induction mediated by JAK1/2-STAT1/3 and CREB contributes to bioenergetic improvements and protective effects against mitochondrial dysfunction in cortical neurons. Biochim Biophys Acta 2015; 1853: 2306-2325.

41 Ikeda Y, Sciarretta S, Nagarajan N, Rubattu S, Volpe M, Frati G et al. New insights into the role of mitochondrial dynamics and autophagy during oxidative stress and aging in the heart. Oxid Med Cell Longev 2014; 2014: 210934.
42 Gritsina G, Xiao F, O'Brien SW, Gabbasov R, Maglaty MA, Xu RH et al. Targeted blockade of JAK/STAT3 signaling inhibits ovarian carcinoma growth. Mol Cancer Ther 2015; 14: 1035-1047.

43 Wright AA, Cronin Al, Milne DE, Bookman MA, Burger BA, Cohn DE et al. Use and effectiveness of intraperitoneal chemotherapy for treatment of ovarian cancer. J Clin Oncol 2015; 33: 2841-2847.

44 Buick RN, Pullano R, Trent JM. Comparative properties of five hu man ovarian adenocarcinoma cell lines. Cancer Res 1985; 45: 3668-3676.

45 Hamilton TC, Young RC, McKoy WM, Grotzinger KR, Green JA, Chu EW et al. Characterization of a human ovarian carcinoma cell line (NIH:OVCAR-3) with androgen and estrogen receptors. Cancer Res 1983; 43: 5379-5389.

46 Abedin MJ, Wang D, McDonnell MA, Lehmann U, Kelekar A. Autophagy delays apoptotic death in breast cancer cells following DNA damage. Cell Death Differ 2007; 14: 500-510.

47 O'Donovan TR, O'Sullivan GC, McKenna SL. Induction of autophagy by drugresistant esophageal cancer cells promotes their survival and recovery following treatment with chemotherapeutics. Autophagy 2011; 7: 509-524.

48 Hsu KF, Wu CL, Huang SC, Wu CM, Hsiao JR, Yo YT et al. Cathepsin L mediates resveratrol-induced autophagy and apoptotic cell death in cervical cancer cells. Autophagy 2009; 5: 451-460.

49 Xia SL, Wu ML, Li H, Wang JH, Chen NN, Chen XY et al. CRABP-II- and FABP5-independent responsiveness of human glioblastoma cells to all-trans retinoic acid. Oncotarget 2015; 6: 5889-5902.

50 Zhang H, Chang JT, Guo B, Hansen M, Jia K, Kovács AL et al. Guidelines for monitoring autophagy in Caenorhabditis elegans. Autophagy 2015; 11: 9-27.

51 Lanuti P, Bertagnolo V, Pierdomenico L, Bascelli A, Santavenere E, Alinari L et al. Enhancement of TRAIL cytotoxicity by AG-490 in human ALL cells is characterized by downregulation of CIAP-1 and CIAP-2 through inhibition of Jak2/Stat3. Cell Res 2009; 19: 1079-1089.

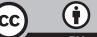

This work is licensed under a Creative Commons Attribution 4.0 International License. The images or other third party material in this article are included in the article's Creative Commons license, unless indicated otherwise in the credit line; if the material is not included under the Creative Commons license, users will need to obtain permission from the license holder to reproduce the material. To view a copy of this license, visit http://creativecommons.org/licenses/ by/4.0/ 